\title{
Feasibility study of in-beam polarization of fluorine
}

\author{
C.D.P. Levy ${ }^{\mathrm{a}, *}$, T.E. Cocolios ${ }^{\mathrm{b}, 1}$, J.A. Behr ${ }^{\mathrm{a}}$, K. Jayamanna ${ }^{\mathrm{a}}$, \\ K. Minamisono ${ }^{\mathrm{a}, 2}$, M.R. Pearson ${ }^{\mathrm{a}}$ \\ ${ }^{\mathrm{a}}$ TRIUMF, 4004 Wesbrook Mall, Vancouver, BC, Canada V6T $2 A 3$ \\ ${ }^{\mathrm{b}}$ Mc Gill University, Montreal, Canada H3A 2 T8
}

Received 10 May 2007; received in revised form 19 June 2007; accepted 8 July 2007

Available online 20 July 2007

\begin{abstract}
The feasibility of in-beam polarization of nuclear spin in $\mathrm{F}^{+}$beams was studied. It was demonstrated that metastable atomic fluorine could be optically pumped on the closed transition $\left(3 \mathrm{~s}^{4} \mathrm{P}_{5 / 2} \rightarrow 3 \mathrm{p}{ }^{4} \mathrm{D}_{7 / 2}\right)$ at $686 \mathrm{~nm}$. The metastable production efficiency in sodium vapor was measured using optical depopulation pumping of the metastable on the $\left(3 \mathrm{~s}^{4} \mathrm{P}_{5 / 2} \rightarrow 3 \mathrm{p}{ }^{4} \mathrm{D}_{5 / 2}\right)$ transition at $678 \mathrm{~nm}$. At low helium density, $0.24(+0.16 /-0.03)$ of the reionized beam was derived from metastables, implying an ion beam polarization of $24 \%$ would be feasible if the metastables were fully polarized. The hyperfine structures of the $3 s{ }^{4} \mathrm{P}_{5 / 2}, 3 p{ }^{4} \mathrm{D}_{5 / 2}$ and $3 p{ }^{4} \mathrm{D}_{7 / 2}$ states in ${ }^{19} \mathrm{~F}$ were measured via laser-induced fluorescence and modulated optical depopulation pumping. Based on these results, a scheme is suggested for polarizing ${ }^{20} \mathrm{~F}$.
\end{abstract}

(C) 2007 Elsevier B.V. All rights reserved.

PACS: $29.27 . \mathrm{Hj} ; 32.10 \mathrm{Fn} ; 39.30 .+\mathrm{w} ; 34.50 . \mathrm{Fa}$

Keywords: Radioactive polarized beam; Metastable fluorine; Hyperfine structure

\section{Introduction}

Closed electronic transitions that cycle between two atomic energy levels are used in optical trapping and cooling, and in producing electronic and nuclear spin polarization by means of optical pumping with circularly polarized light. The majority of cases involve alkali-metal atoms, in which the strong ground state $D_{1}$ and $D_{2}$ transitions are within easy reach of commonly available lasers. Polarization of $\mathrm{Be}^{+}$ions has been achieved with a relatively low power frequency-doubled dye laser [1]. In elements where transitions from the ground state are too high in energy to be easily accessed by lasers, metastable atoms formed in collisions may serve as the lower level. The latter technique has been applied to the trapping of metastable neon atoms [2]. Metastable levels can also be

\footnotetext{
*Corresponding author. Tel.: + 16042227424 ; fax: + 16042221074 .

E-mail address: levy@triumf.ca (C.D.P. Levy).

${ }^{1}$ Now at IKS, K.U. Leuven, Belgium.

${ }^{2}$ Now at NSCL, Michigan State University, East Lansing, USA.
}

populated or depopulated by optical pumping on open transitions. Very sensitive versions of collinear-laser fastbeam spectroscopy are based on the fact that collisional ionization or neutralization cross-sections are different for metastable and ground state levels. Optical pumping leads (in a subsequent gas or vapor cell) to a change in ionization or neutralization efficiency that is observable using particle detection techniques. High-resolution spectroscopy of raregas atoms, alkaline-earth ions, and mercury and thallium atoms [3] has been carried out in this way.

The polarized radioactive beam facility at TRIUMFISAC presently produces low energy beams of lithium and sodium isotopes with nuclear spin polarizations of typically $60 \%$ [4]. Ground state atoms, created from an incident ion beam in a sodium vapor charge exchange cell, are polarized in-beam by optically pumping the $D_{1}$ transition with circularly polarized anti-collinear laser light in a small longitudinal magnetic field. The polarized fast atomic beam is then reionized in a helium gas target and transported to the user. Here we report on the feasibility of polarizing $\mathrm{F}^{+}$ beams in a similar fashion. The motivation for this work 
was to develop polarized radioactive fluorine beams at TRIUMF-ISAC for the second-class current search in the mass $A=20$ system [5] and for $\beta$-NMR measurements of nuclear moments in neutron rich fluorine isotopes for nuclear structure investigations and other studies.

Although optical pumping of the fluorine atomic ground state is not practicable due to the short wavelength light required, we identified the metastable atomic state $3 \mathrm{~s}{ }^{4} \mathrm{P}_{5 / 2}$ $\left(102406 \mathrm{~cm}^{-1}\right.$ above the ground state; published lifetimes $7.3 \pm 0.5 \mu \mathrm{s}$ [6], $3.7 \pm 0.5 \mu \mathrm{s}$ [7]) as a candidate for optical pumping on the closed transition $3 s{ }^{4} \mathrm{P}_{5 / 2} \rightarrow 3 p{ }^{4} \mathrm{D}_{7 / 2}$ at $686 \mathrm{~nm}$. We were led to consider this transition by a suggestion that it be used for laser cooling [7]. A term diagram of the $3 \mathrm{~s}^{4} \mathrm{P}_{J} \rightarrow 3 \mathrm{p}^{4} \mathrm{D}_{J^{\prime}}$ fine structure transition multiplet is shown in Fig. 1. (Throughout this paper, unprimed angular momentum quantum numbers $(J, F)$ refer to the lower-energy level and primed quantities $\left(J^{\prime}, F^{\prime}\right)$ refer to the upper level.) The viability of this scheme depends on the metastables forming a significant fraction of the neutralized beam, and being in fact part of a nearly closed transition and long-lived enough to survive the $2 \mu \mathrm{s}$ transit time between the sodium and helium cells.

The metastable fraction was measured using optical depopulation pumping of the metastable on the $3 \mathrm{~s}{ }^{4} \mathrm{P}_{5 / 2} \rightarrow$ $3 \mathrm{p}^{4} \mathrm{D}_{5 / 2}$ transition at $678 \mathrm{~nm}$. We were not able to measure directly the polarization of the stable ${ }^{19} \mathrm{~F}$ beam; our polarization measurement method relies on detecting the spatial asymmetry of beta emission by radioactive isotopes. In the course of the study the hyperfine structure (hfs) of the $3 s^{4} \mathrm{P}_{5 / 2}, 3 p{ }^{4} \mathrm{D}_{5 / 2}$ and $3 p{ }^{4} \mathrm{D}_{7 / 2}$ states in ${ }^{19} \mathrm{~F}$ (nuclear spin $I=\frac{1}{2}$ ) were measured using laserinduced fluorescence and modulated optical depopulation pumping.

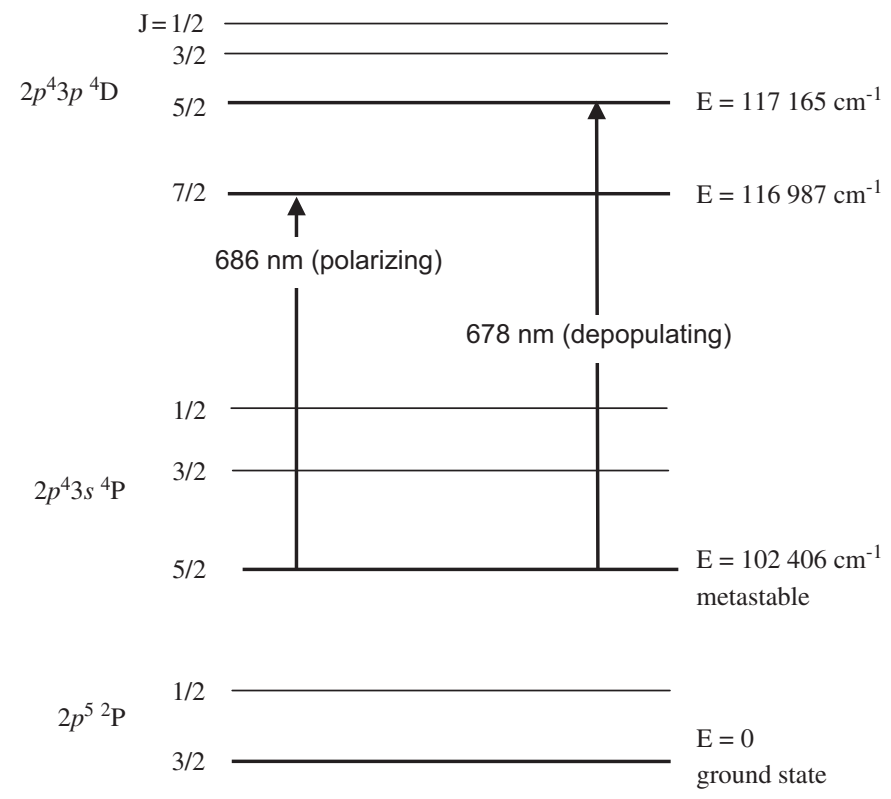

Fig. 1. Transitions and fine structure of fluorine atomic energy levels relevant to this work.

\section{Experimental methods}

The experimental apparatus used to do collinear laser spectroscopy on metastable fluorine atoms is shown schematically in Fig. 2. A microwave ion source using $\mathrm{CF}_{4}$ as the feed gas produced an ion beam that was accelerated to an energy of $40.8 \mathrm{keV}$ and directed through a mass separator magnet that selected ${ }^{19} \mathrm{~F}^{+}$ions. The ${ }^{19} \mathrm{~F}^{+}$ beam was transported $18 \mathrm{~m}$ by means of electrostatic steering and focusing elements to the interaction region, where it was focused by electrostatic quadrupoles (having a focal length of approximately $2 \mathrm{~m}$ ) through the $12 \mathrm{~mm}$ diameter sodium vapor cell aperture and a downstream $8 \mathrm{~mm}$ diameter aperture just upstream of a helium gas reionization cell. The intensity of the beam at the entrance to the neutralization cell was in the range $10-100 \mathrm{nA}$. The ion beam was neutralized with typically $40-50 \%$ efficiency by charge exchange in the sodium vapor cell, with negligible effect on the transverse beam emittance. Electrostatic deflector plates between the sodium and helium cells swept out of the beam all the ions that passed unneutralized through the sodium cell. The neutralization efficiency was determined by observing the drop in positive ion current at Faraday cup FC1 (see Fig. 2) as the cell temperature was raised. The polarity of the deflection plates was reversed temporarily to check for the presence of $\mathrm{F}^{-}$ions. No negative current was observed.

Some fraction of the fast neutralized beam consisted of excited fluorine atoms in the metastable $3 \mathrm{~s}^{4} \mathrm{P}_{5 / 2}$ state. The metastable atoms exiting the sodium cell traveled anticollinearly with a fixed frequency cw laser beam over the $1.9 \mathrm{~m}$ distance between the sodium and helium cells. The effective laser frequency was tuned by Doppler shifting it into resonance with the $3 \mathrm{~s}^{4} \mathrm{P}_{J} \rightarrow 3 \mathrm{p}^{4} \mathrm{D}_{J^{\prime}}$ transitions by a variable electrical potential applied to post-acceleration electrodes and the sodium cell. The potential was measured with a precision voltmeter. The annular electrodes in front of the sodium cell produced a smooth electric field that preserved the spatial beam quality. A good overlap was obtained between the converging fast atomic beam and the 2-3 mrad (full angle) diverging laser beam.

A cw ring DCM dye laser pumped by an argon-ion laser was used in these studies. The dye laser was actively

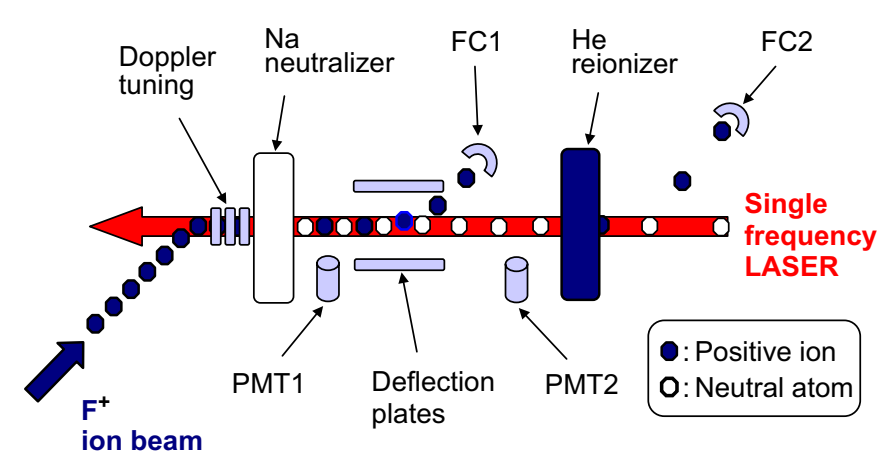

Fig. 2. Sketch of experimental apparatus. 
stabilized and had a bandwidth of approximately $1 \mathrm{MHz}$. A sample beam from the dye laser and a frequencystabilized helium-neon laser were simultaneously monitored by a hermetically sealed, temperature stabilized, $300 \mathrm{MHz}$ free spectral range, scanning Fabry-Perot interferometer. Computer-controlled feedback to the dye laser kept the interference pattern fringe separation constant. This locked the dye laser frequency to the helium-neon reference, which was stable to several $\mathrm{MHz} / \mathrm{day}$. The dye laser's absolute frequency was measured with an accuracy of approximately $300 \mathrm{MHz}$ by a commercial wavemeter containing four neon filled, temperature-stabilized Fizeau interferometers, calibrated using the frequency stabilized helium-neon laser.

The hfs of the closed transition $3 s{ }^{4} \mathrm{P}_{5 / 2} \rightarrow 3 p{ }^{4} \mathrm{D}_{7 / 2}$ was obtained by measuring laser-induced fluorescence. The fluorescence was detected by a cooled photon counter that viewed the beam normal to both the beam direction and the plane of polarization of the vertically polarized light. The field of view of the detection system was approximately an $8 \mathrm{~mm}$ diameter ball centered on the beam axis. The laser intensity was approximately $50 \mathrm{~mW} / \mathrm{cm}^{2}$. Stray laser light was removed using a narrow band interference filter that transmitted only the fluorescence, which was $1.5 \mathrm{~nm}$ shorter in wavelength than the laser light as seen by the detector. Detectors PMT1 and PMT2 were available (see Fig. 2), 40 and $135 \mathrm{~cm}$ downstream of the center of the sodium cell, respectively. Spectra were recorded by sweeping the sodium cell voltage in steps of $0.6 \mathrm{~V}-$ equivalent to a change in Doppler-shifted laser frequency of $7 \mathrm{MHz}$ - and integrating the photon counts for $1 \mathrm{~s}$. A typical scan time was less than 10 min and scans were done in both directions.

The hfs of the open $3 \mathrm{~s}{ }^{4} \mathrm{P}_{5 / 2} \rightarrow 4 \mathrm{p}{ }^{4} \mathrm{D}_{5 / 2}$ transition was recorded by detecting changes in reionized beam current as the sodium cell voltage was swept. As above, the fluorine beam was partially neutralized in the sodium cell and the metastable ${ }^{4} \mathrm{P}_{5 / 2}$ atoms were excited by laser light resonant with the relevant transition. Unlike the case of the closed transition, the metastable state was quickly depopulated on resonance, since the ${ }^{4} \mathrm{D}_{5 / 2}$ state could decay to the ${ }^{4} \mathrm{P}_{3 / 2}$ state and from there to the ground state. The beam then passed through the helium gas cell where it was partially reionized. The ion beam exiting the helium cell was separated from the remaining fast atomic beam by an electrostatic bend and collected by Faraday cup FC2. Fig. 3 shows the reionization efficiency dependence on the helium flow rate, corrected for the loss of collection efficiency of the relatively small diameter FC2 due to elastic scattering by the helium. Metastable atoms had a higher ionization cross-section than ground state atoms, and therefore the current recorded at FC2 was smaller when the laser light was resonant with a hyperfine transition and had depleted the metastable population. The laser was chopped at a frequency of $1.7 \mathrm{kHz}$ while the voltage was swept. The current modulation at FC2 was converted to a DC voltage by a phase locked amplifier and

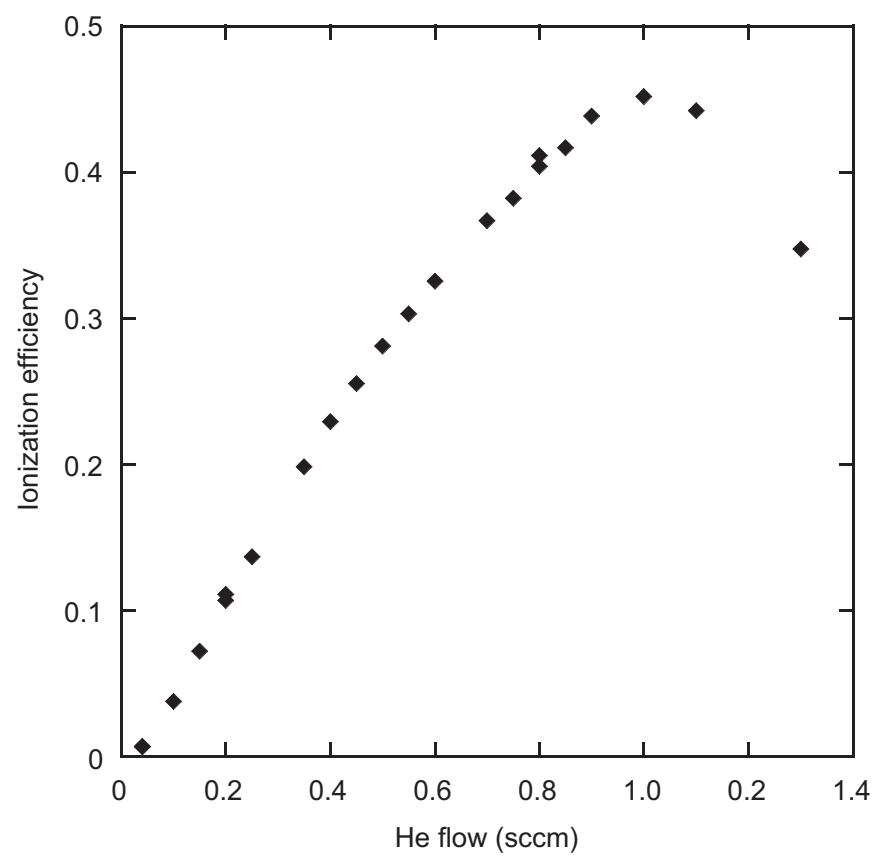

Fig. 3. Fluorine beam reionization efficiency in helium cell. The flow rate is given in units of standard cubic centimeters per minute.

then converted to pulses by a voltage-to-frequency converter and sent to a scaler.

The currents at FC2 with the unchopped laser "on" and "off" were then compared at different laser powers and helium densities, while tuned to the peak of the strongest hyperfine transition. This permitted us to estimate the fraction of the beam in the metastable state at the entrance to the helium cell, and the fraction of ion beam derived from metastable atoms after preferential reionization, as discussed in the next section.

\section{Results and discussion}

\section{1. ${ }^{4} P_{5 / 2} \rightarrow{ }^{4} D_{7 / 2}$ transition}

A spectrum of the cycling ${ }^{4} \mathrm{P}_{5 / 2} \rightarrow{ }^{4} \mathrm{D}_{7 / 2}$ transition observed at PMT1 is shown in Fig. 4. The three allowed hyperfine components were identified on the basis of their relative intensities. In Fig. 4 each transition is labeled with its relative transition strength, calculated using angular momentum coupling coefficients. Although the calculated values differed from the observed fluorescence intensities due to optical pumping and saturation effects, line identification was unambiguous. We note that our line assignments disagree with those of Shimizu [6], who did not observe the $F \rightarrow F^{\prime}=3 \rightarrow 3$ transition. The peaks were fitted to Lorentzian profiles using commercial software. The differences between Lorentzian, Gaussian and Voigt profile fits were insignificant at our level of accuracy. No significant effect was observed whether the peaks were fitted singly (4 parameter fit) or in pairs (7 parameter fit), or if the highest count rate data were discarded. Light shifts 


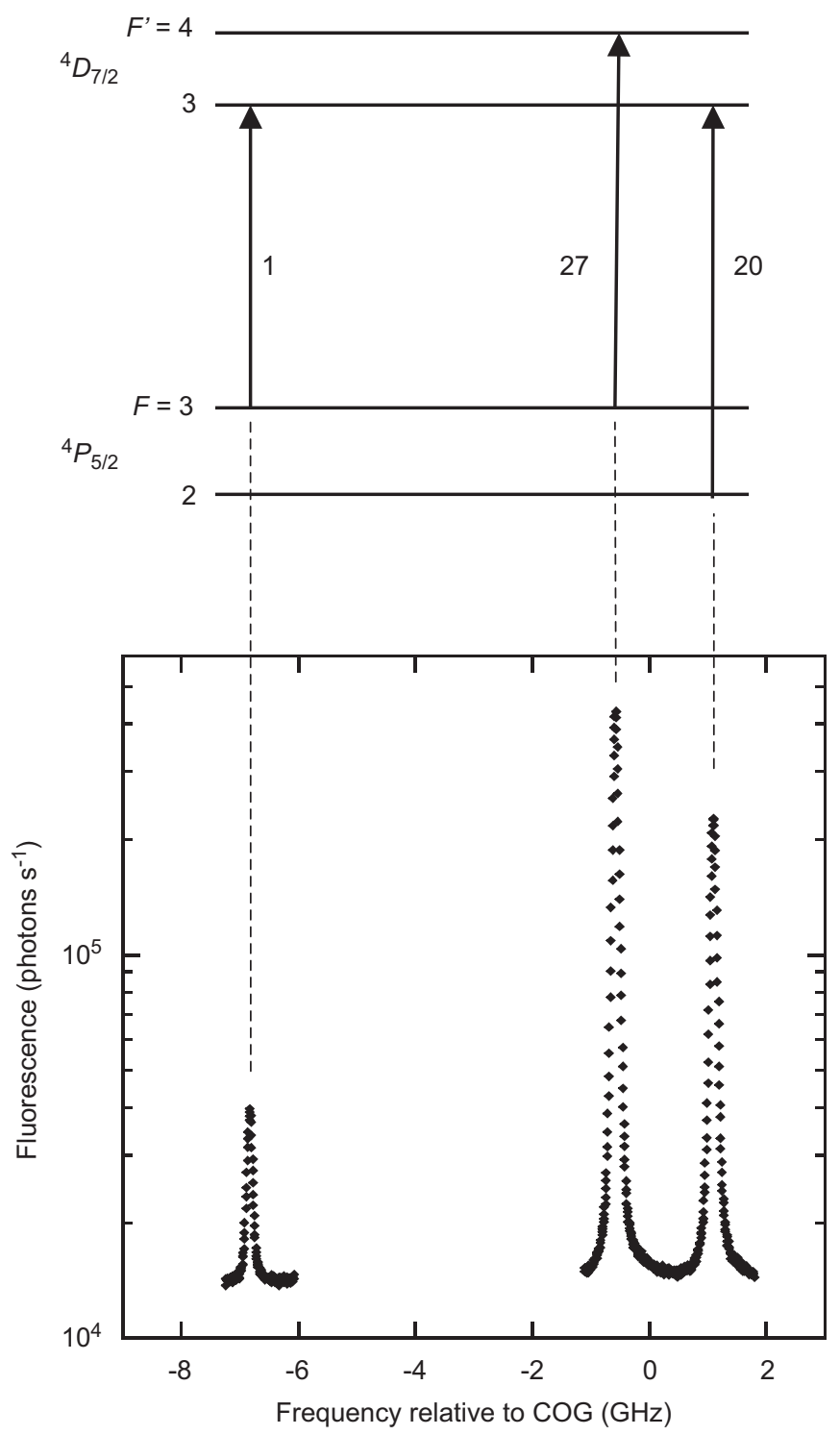

Fig. 4. Hyperfine spectrum of the ${ }^{4} \mathrm{P}_{5 / 2} \rightarrow{ }^{4} \mathrm{D}_{7 / 2}$ transition in ${ }^{19} \mathrm{~F}$ obtained from laser-induced fluorescence. COG frequency $v_{\mathrm{COG}}=14581.684 \mathrm{~cm}^{-1}$ [8]. Note the logarithmic scale.

were insignificant, as were shifts due to changes in beam emittance as the spectrum was scanned. The splitting was calculated separately for each scan, and the results averaged. The statistical error in the average was $0.5 \mathrm{MHz}$.

The Doppler-shifted laser frequency, $v_{\text {Doppler, was }}$ calculated from the beam energy, $E$, using

$v_{\text {Doppler }}=v_{\text {laser }} \sqrt{\frac{1+\beta}{1-\beta}}$

where $v_{\text {laser }}$ is the fixed laser frequency, $\beta=v / c, v=$ $(2 E / m)^{1 / 2}, v$ is the atomic beam speed, $c$ is the speed of light and $m$ is the mass of the ${ }^{19} \mathrm{~F}$ atom. At $41 \mathrm{keV}$ beam energy the nonrelativistic expression for $v$ is sufficiently accurate. Table 1 lists the hyperfine splittings and compares them with previous values from Lidén [8]. Most of our error was
Table 1

Hyperfine data on atomic ${ }^{19} \mathrm{~F}$ excited states

\begin{tabular}{lllll}
\hline & \multicolumn{2}{l}{ Hyperfine splitting $(\mathrm{MHz})$} & & \multicolumn{2}{l}{ Hyperfine constant $A(\mathrm{MHz})$} \\
\cline { 2 - 3 } & This work & Ref. [8] & & This work \\
\hline $3 \mathrm{~s}^{4} \mathrm{P}_{5 / 2}$ & $7930(3)$ & $7760(150)$ & $+2643(1)$ \\
$3 \mathrm{p}{ }^{4} \mathrm{D}_{5 / 2}$ & $3443(4)$ & $3240(150)$ & & $+1148(1)$ \\
$3 \mathrm{p}{ }^{4} \mathrm{D}_{7 / 2}$ & $6256(3)$ & $6240(150)$ & & $+1564(1)$ \\
\hline
\end{tabular}

from systematic $\sim 30 \mathrm{eV}$ uncertainty in $E$. This in turn arose from the uncertainties in $v_{\text {laser }}$ and in the transition-multiplet frequency center-of-gravity, $v_{\mathrm{COG}}=$ $14581.684 \mathrm{~cm}^{-1}$ [8], used to calibrate $E$. Calibration was done by first determining the sodium cell voltage COG of the observed peaks, using theoretical intensities for the hyperfine transitions because the measured intensities were influenced by optical pumping effects. The relation between $E$ and $v_{\text {Doppler }}$ was almost linear over the transition multiplet, so to a very good approximation the voltage COG was in the same position as $v_{\mathrm{COG}}$ in relation to the peaks. The beam energy at the voltage COG was then calibrated by setting $v_{\text {Doppler }}=v_{\mathrm{COG}}$ in Eq. (1). We trusted Lidén's claims for $v_{\mathrm{COG}}$ accuracy $(150 \mathrm{MHz})$, since he clearly resolved the $\mathrm{hfs}$ of this transition. A recent measurement of the ${ }^{2} \mathrm{P}_{1 / 2} \rightarrow{ }^{2} \mathrm{D}_{3 / 2}$ COG transition frequency [9] differed from Lidén's value by $550 \mathrm{MHz}$, but he did not resolve the hfs in that transition. Error in measuring the hyperfine splitting leads directly to error in the calculated $v_{\mathrm{COG}}$ when the latter is based on a measured frequency of one of the components.

Observation of fluorescence well downstream of the point of origin of the metastables showed qualitatively that the overall transition was closed or nearly so. Hyperfine pumping from $F=2 \rightarrow 3$ within the ${ }^{4} \mathrm{P}_{5 / 2}$ multiplet was observed at PMT1 as a decrease in the fluorescence from the transition $F \rightarrow F^{\prime}=2 \rightarrow 3$ relative to the closed transition $F \rightarrow F^{\prime}=3 \rightarrow 4$ as the laser power was increased, as shown in Fig. 5.

The variation of fluorescence signal ratio at PMT2 between circularly and linearly polarized pump light on the $F \rightarrow F^{\prime}=3 \rightarrow 4$ transition was also measured with varying laser power, as shown in Fig. 6. In that case, a longitudinal magnetic field of $\sim 10$ gauss was applied over the optical pumping region. The laser bandwidth was broadened to approximately $100 \mathrm{MHz}$ by passing the laser beam through 19 and $28 \mathrm{MHz}$ electro-optic modulators (EOMs) in series, thus producing multiple sidebands at 9 and $10 \mathrm{MHz}$ intervals, in order to address the entire Doppler-broadened population in the $F=3$ state. The observed ratio was consistent with optical pumping by circular light of the initially unpolarized $F=3$ state into stretched magnetic substates. The total transition strength increased as the substates became more stretched on average, which accounted for the increase in the ratio as the $F=3$ state became more polarized with rising laser power. 


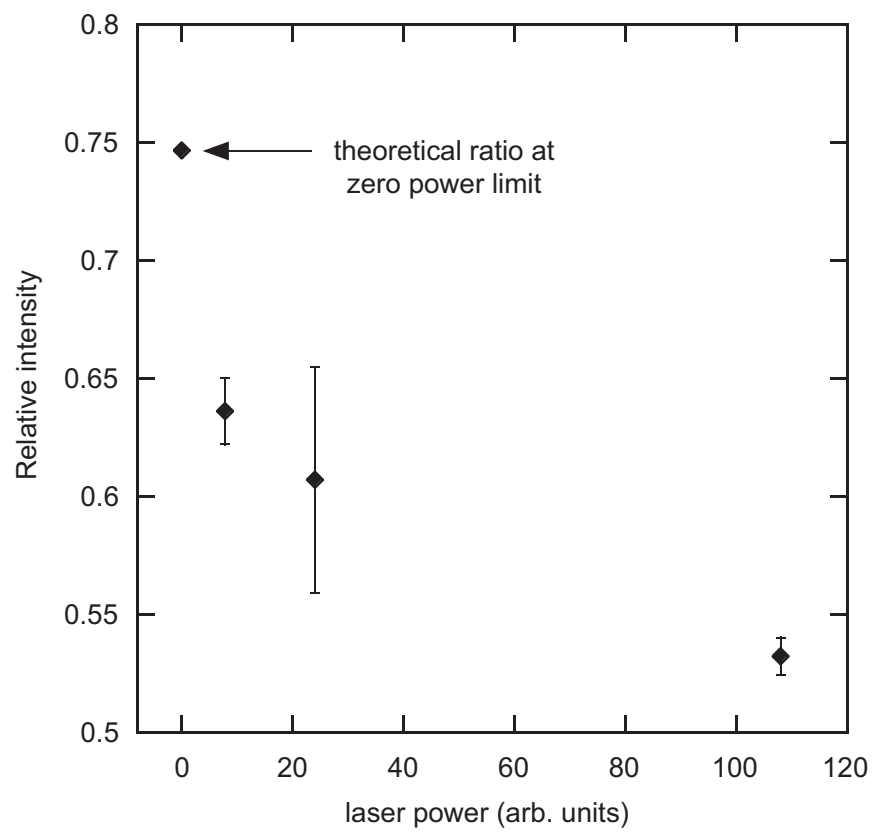

Fig. 5. Variation with laser power of the fluorescence intensity observed during excitation of the $F \rightarrow F^{\prime}=2 \rightarrow 3$ transition compared to excitation of the $F \rightarrow F^{\prime}=3 \rightarrow 4$ transition in the ${ }^{4} \mathrm{P}_{5 / 2} \rightarrow{ }^{4} \mathrm{D}_{7 / 2}$ transition multiplet.

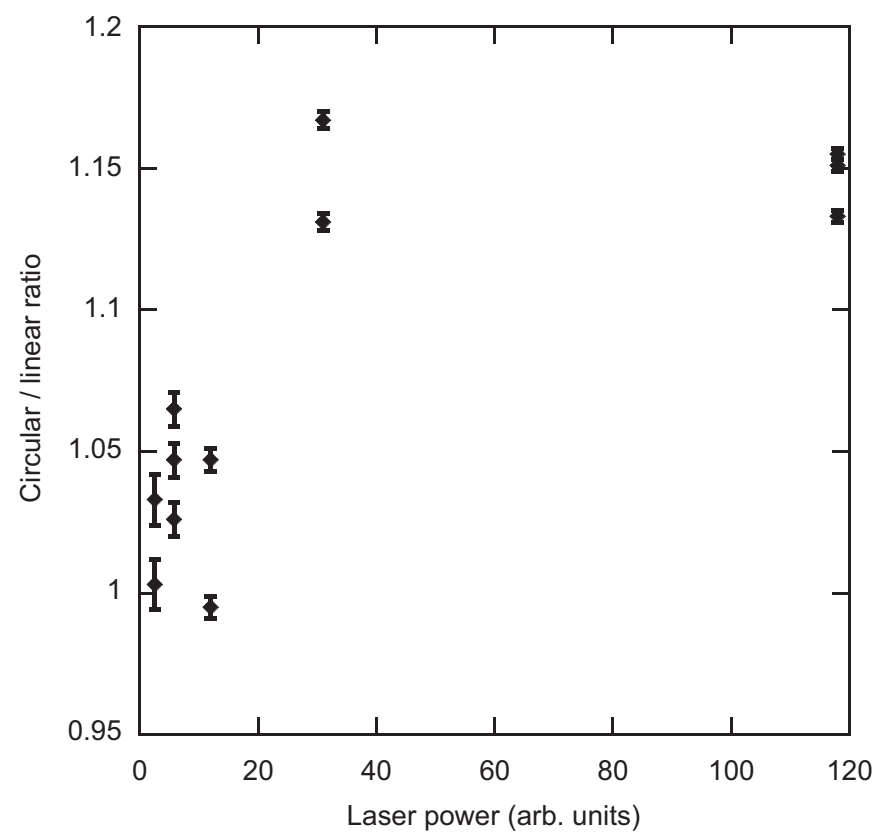

Fig. 6. The ratio between fluorescence signals observed with circular and linear light, as a function of laser power on a closed hyperfine transition within the ${ }^{4} \mathrm{P}_{5 / 2} \rightarrow{ }^{4} \mathrm{D}_{7 / 2}$ transition multiplet of ${ }^{19} \mathrm{~F}$. The error bars indicate the known sources of error.

\section{2. ${ }^{4} P_{5 / 2} \rightarrow{ }^{4} D_{5 / 2}$ transition and metastable yield}

A spectrum obtained by metastable depopulation pumping is shown in Fig. 7. The scanning power supply had insufficient range to permit the observation of the whole hfs in a single sweep. The spectrum shown is the

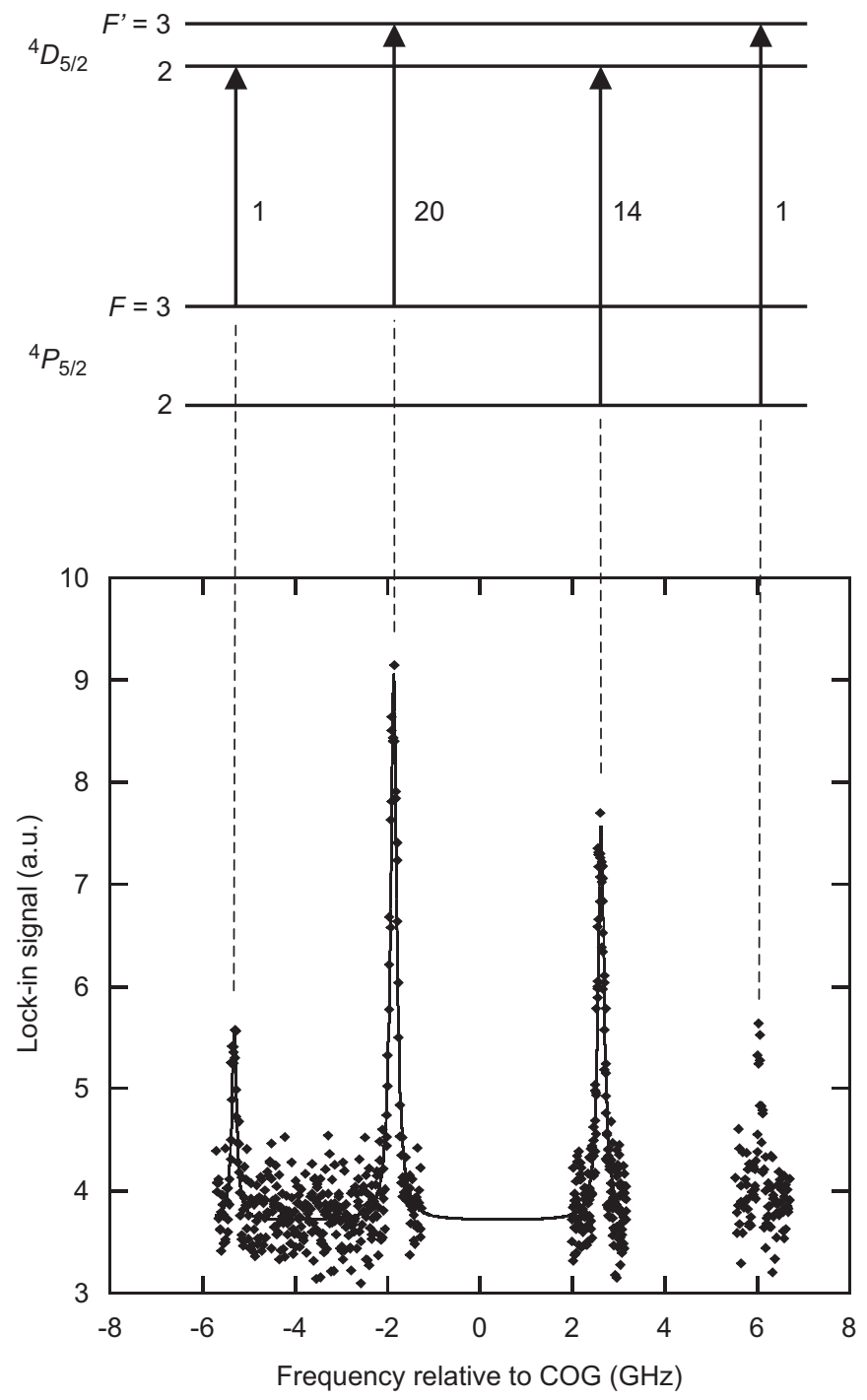

Fig. 7. Hyperfine spectrum of the ${ }^{4} \mathrm{P}_{5 / 2} \rightarrow{ }^{4} \mathrm{D}_{5 / 2}$ transition in ${ }^{19} \mathrm{~F}$ obtained from optical depopulation pumping of the metastable state. COG frequency $v_{\mathrm{COG}}=14758.296 \mathrm{~cm}^{-1}$ [8]. Because of the large splitting, only three lines could be scanned at a time; the curve is a triple Lorentzian fit to one of those scans.

combination of two sweeps with different DC offsets taken close together in time to show the complete structure. Four hyperfine transitions were observed and were identified on the basis of their relative intensities and the previously determined ${ }^{4} \mathrm{P}_{5 / 2}$ state hyperfine splitting and ordering. As in Fig. 4, the calculated optical transition strengths used as a guide in identifying the lines are shown. The onresonance signals are opposite in sign to the change in current due to the setting of the phase-locked amplifier. The splitting of the ${ }^{4} \mathrm{D}_{5 / 2}$ level is included in Table 1 . Statistical error dominates in this case.

Spectra with greatly improved signal-to-noise ratios were taken during a later run with a more stable ion source current and a broadened laser bandwidth, but could not be used to measure hyperfine splittings due to an uncalibrated laser wavemeter. However, the metastable yield was estimated under the better noise conditions, as follows. 


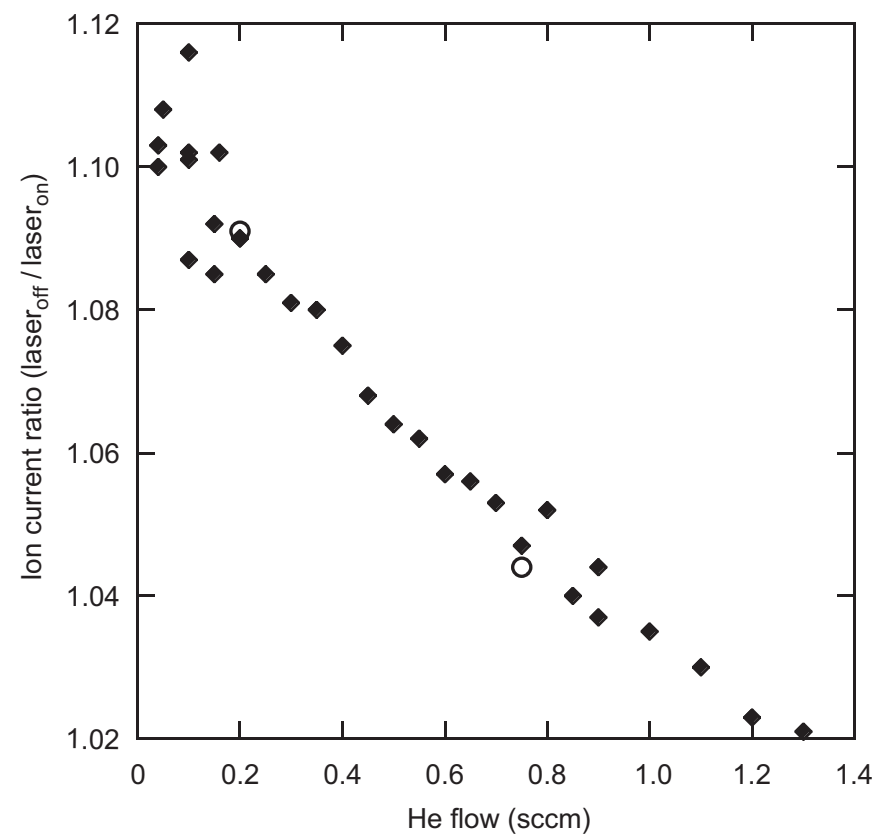

Fig. 8. The ratio of reionized beam currents observed with laser depopulation pumping off and on, tuned to the strongest hyperfine transition in the ${ }^{4} \mathrm{P}_{5 / 2} \rightarrow{ }^{4} \mathrm{D}_{5 / 2}$ multiplet in ${ }^{19} \mathrm{~F}$, as a function of helium flow rate.

The laser frequency was Doppler tuned to the center of the $F \rightarrow F^{\prime}=3 \rightarrow 3$ transition and broadened with the EOMs. The ion current at FC2 was then simply recorded with the laser "on" and "off". Fig. 8 shows the dependence of the ion current ratio $R=($ laser "off" $) /$ (laser "on") on helium flow rate. The two unfilled data points were measured at half the nominal laser power, and showed no change in current modulation, indicating that the $F=3$ level was completely emptied by optical pumping.

The data were interpreted in the following way. The ion current detected at FC2 with the laser blocked was given by

$I_{\text {off }}=I\left[f^{*}\left(1-\mathrm{e}^{-\rho k}\right)+\left(1-f^{*}\right)\left(1-\mathrm{e}^{-k}\right)\right]$

where $I$ is a constant proportional to the source current, $f^{*}$ is the metastable fraction of the atomic fluorine beam at the entrance to the helium cell, $k$ is an ionization constant proportional to the helium flow rate, and $\rho$ is the ratio of metastable to ground state ionization cross-sections. When the laser was resonant with the $F \rightarrow F=3 \rightarrow 3$ transition, the ion current was given by

$I_{\text {on }}=I\left[0.428 f^{*}\left(1-\mathrm{e}^{-\rho k}\right)+\left(1-0.428 f^{*}\right)\left(1-\mathrm{e}^{-k}\right)\right]$.

The factor 0.428 is the fraction of metastables left in the $F=2$ state after the depopulation of $F=3$ by hyperfine pumping. The assumed initial statistical population for the $F=2$ state is 0.417 . The upper ${ }^{4} \mathrm{D}_{5 / 2}$ state decays to either $3 \mathrm{~s}{ }^{4} \mathrm{P}_{5 / 2}$, 3s ${ }^{4} \mathrm{P}_{3 / 2}$, or $3 \mathrm{~s}{ }^{2} \mathrm{P}_{3 / 2}$, with measured relative strengths $7: 15: 1[8]$, which we correct to $6.8: 15: 1$ for the $F=3$ state alone. The strengths in Fig. 7 show that only $1 / 21$ of the decay back to $3 \mathrm{~s}{ }^{4} \mathrm{P}_{5 / 2}$ is to the $F=2$ state. So the $F=2$ fraction rises from 0.417 to only $0.428( \pm 0.002)$, and it reaches this final value within just a few depumping cycles. The stated error is mainly due to uncertainty in the measured line strengths, somewhat arbitrarily assumed to be $10 \%$. It does not include any systematic error in the initial value 0.417 .

As the helium density tends to zero, Eqs. (2) and (3) reduce to

$f^{*}=\frac{R_{0}-1}{\left(1-0.428 R_{0}\right)(\rho-1)}$

where $R_{0}=I_{\text {off }} / I_{\text {on }}$ as the helium density tends to zero. The ratio $R_{0}$ was measured to be $1.105 \pm 0.005$ (see Fig. 8). The preferential ionization factor $\rho=3.4$ was estimated using an approximate method for calculating absolute ionization cross-sections [10], giving the result $f^{*}=0.083$. Note that $f^{*}$ is the fraction remaining after decay losses in transit between the sodium and helium cells and is of course independent of $\rho ; R_{0}$ depends on $\rho$ and $f^{*}$.

The fraction of reionized beam derived from the metastables, $F^{*}$, is of most interest since that equals the maximum possible beam polarization. Under the condition of no laser pumping

$F^{*}=\frac{I f^{*}\left(1-\mathrm{e}^{-\rho k}\right)}{I_{\mathrm{off}}}$

where the numerator is the portion of reionized beam derived from metastables and the denominator is the total reionized beam. At low $k$, where $F^{*}$ is largest, this reduces to

$F_{0}^{*}=\frac{f^{*} \rho}{1+f^{*}(\rho-1)}$

We can eliminate $f^{*}$ from the above expression using Eq. (4) to obtain

$F_{0}^{*}=\frac{\left(R_{0}-1\right) \rho}{0.572 R_{0}(\rho-1)}$.

A conservative estimate of the accuracy of $\rho$ is $50 \%$. This gives the result $F_{0}^{*}=0.24(+0.16 /-0.03)$. In the operational case of strong optical pumping at $686 \mathrm{~nm}, \rho$ and hence $F_{0}^{*}$ would be increased by further enhanced ionization of population in the highest ${ }^{4} \mathrm{D}_{7 / 2}$ state. In addition, decay of the metastable state would be reduced because of time spent in the ${ }^{4} \mathrm{D}_{7 / 2}$ state, thus increasing $f^{*}$. Omitting the helium cell and instead photoionizing the ${ }^{4} \mathrm{D}_{7 / 2}$ state with laser light at $424 \mathrm{~nm}$ or shorter would be a way to achieve $F^{*}=1$, albeit with reduced ionization efficiency.

\section{Application to other isotopes}

We wish to polarize ${ }^{20} \mathrm{~F}(I=2)$ and other radioactive fluorine isotopes. One needs to know the hfs before the laser system can be designed, since optical pumping of most of the $(2 I+1){ }^{4} \mathrm{P}_{5 / 2}$ hyperfine levels is required in order to achieve high polarization. The hfs of the radioactive isotopes can be approximately calculated from the ${ }^{19} \mathrm{~F}$ data as follows. ${ }^{19} \mathrm{~F}\left(I=\frac{1}{2}\right)$ has no nuclear electric 


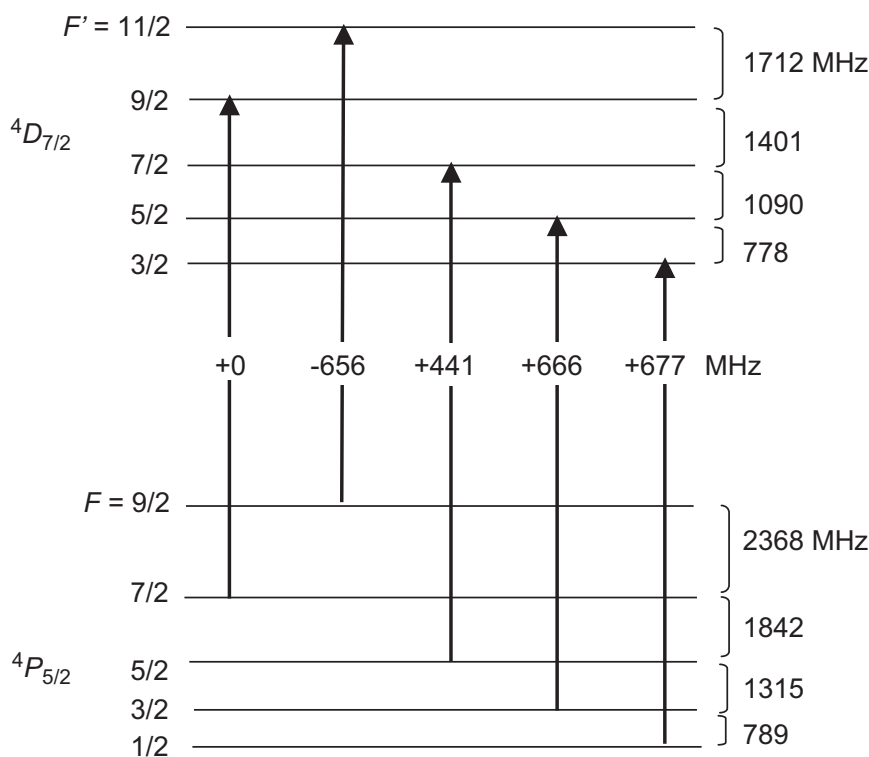

Fig. 9. Calculated hfs of the ${ }^{4} \mathrm{P}_{5 / 2} \rightarrow{ }^{4} \mathrm{D}_{7 / 2}$ transition in ${ }^{20} \mathrm{~F}$, showing the splittings and suitable transitions that must all be optically pumped to fully polarize the metastable atom. The transition labels are the frequency offsets $(\mathrm{MHz})$ to the laser frequency in the suggested laser pumping scheme.

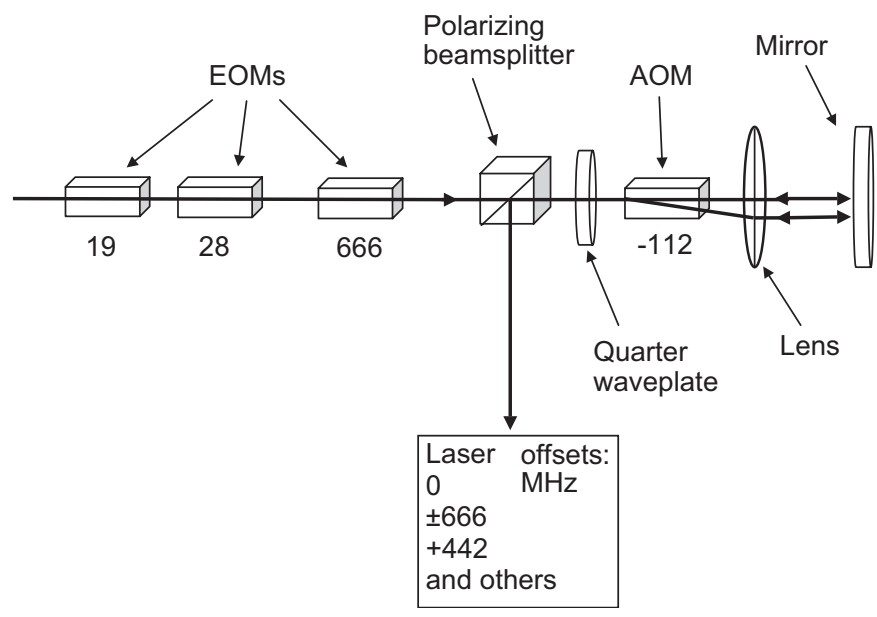

Fig. 10. Optical arrangement for pumping all the hyperfine levels of the ${ }^{4} \mathrm{P}_{5 / 2}$ state in ${ }^{20} \mathrm{~F}$. Values under EOMs and AOM are first-order sideband frequencies $(\mathrm{MHz})$.

quadrupole moment, so the hyperfine magnetic dipole constants $A$ for the fluorine ${ }^{4} \mathrm{P}_{5 / 2}$ and ${ }^{4} \mathrm{D}_{7 / 2}$ states are accurately determined from the measured hfs. The electronic magnetic fields at the nucleus for the two states are calculated by combining the $A$ values with the known ${ }^{19} \mathrm{~F}$ nuclear magnetic dipole moment [11]. From this the hfs due to the magnetic dipole interaction can be calculated for any fluorine isotope for which $I$ and the nuclear magnetic moment are known. Fig. 9 shows the hfs for the ${ }^{4} \mathrm{P}_{5 / 2} \rightarrow{ }^{4} \mathrm{D}_{7 / 2}$ transition in ${ }^{20} \mathrm{~F}(I=2)$ and the frequency differences between transitions suitable for pumping the ${ }^{4} \mathrm{P}_{5 / 2}$ hyperfine levels, neglecting electric quadrupole shifts. A simple upper limit on the $\mathrm{B}$ coefficients for ${ }^{20} \mathrm{~F}$, obtained by scaling the measured $\mathrm{B}$ coefficients for the $4 \mathrm{~s}^{4} \mathrm{P}_{5 / 2} \rightarrow$ $4 \mathrm{p}^{4} \mathrm{D}_{J^{\prime}}$ transitions in ${ }^{35} \mathrm{Cl}$ [12] by the nuclear quadrupole moments [11], implies that the quadrupole shifts in all the listed transitions are less than $5 \mathrm{MHz}$.

If the electric quadrupole shifts are small compared to the $100 \mathrm{MHz}$ bandwidth of the EOM-broadened laser, then an efficient laser scheme is easily devised for ${ }^{20} \mathrm{~F}$. Broadened laser light tuned to the $F \rightarrow F^{\prime}=7 / 2 \rightarrow 9 / 2$ transition and passed through a $666 \mathrm{MHz}$ EOM will optically pump the $F=1 / 2,3 / 2,7 / 2$ and $9 / 2$ states, which comprise $80 \%$ of the population in the ${ }^{4} \mathrm{P}_{5 / 2}$ state statistically. Given the large amounts of laser power available at $686 \mathrm{~nm}$, it might be advantageous to double pass the light through a $-112 \mathrm{MHz}$ acousto-optic modulator as shown in Fig. 10, so as to pump the remaining $F=5 / 2$ state as well.

\section{Conclusion}

We have shown that useful, perhaps high, $\mathrm{F}^{+}$beam polarization is possible using the metastable state $3 p{ }^{4} \mathrm{P}_{5 / 2}$ as the lower level of a cycling transition. We also have measured the hfs of three excited states of atomic ${ }^{19} \mathrm{~F}$ using fast-beam collinear laser spectroscopy, and our results agree with earlier much less accurate measurements, adding to the sparse data available on fluorine hyperfine structure.

\section{Acknowledgment}

This work was financed by TRIUMF, which receives federal funding via a contribution agreement with the National Research Council of Canada.

\section{References}

[1] W. Geithner, S. Kappertz, M. Keim, P. Lievens, R. Neugart, L. Vermeeren, S. Wilbert, V.N. Fedoseyev, U. Köster, V.I. Mishin, V. Sebastian, ISOLDE Collaboration, Phys. Rev. Lett. 83 (1999) 3792.

[2] F. Shimizu, K. Shimizu, H. Takuma, Chem. Phys. 145 (1990) 327.

[3] P. Lievens, E. Arnold, W. Borchers, F. Buchinger, U. Georg, A. Klein, W. Neu, R. Neugart, M. Neuroth, C. Schultz, R.E. Silverans, L. Vermeeren, the ISOLDE Collaboration, Nucl. Instr. and Meth. B 70 (1992) 532 (and references therein).

[4] C.D.P. Levy, R. Baartman, J.A. Behr, R.F. Kiefl, M. Pearson, R. Poutissou, A. Hatakeyama, Y. Hirayama, Nucl. Phys. A 746 (2004) 206c.

[5] K. Minamisono, K. Matsuta, T. Minamisono, C.D.P. Levy, T. Nagatomo, M. Ogura, T. Sumikama, J.A. Behr, K.P. Jackson, H. Fujiwara, M. Mihara, M. Fukuda, Hyperfine Interactions 159 (2004) 265.

[6] M. Shimizu, H. Ohmi, H. Kakiuchi, K. Yasutake, J. Vac. Sci. Technol. A 24 (2006) 2133.

[7] M. Shimizu, K. Yasutake, H. Ohmi, A. Takeuchi, H. Kakiuchi, K. Yoshii, Y. Mori, Appl. Phys. B 72 (2001) 227.

[8] K. Lidén, Arkiv för Fysik 1 (1949) 229.

[9] D.A. Tate, D.N. Aturaliye, Phys. Rev. A 56 (1997) 1844.

[10] O.B. Firsov, Soviet Phys. JETP 36 (1959) 1076.

[11] N.J. Stone, Atom. Data Nucl. Data Tables 90 (2005) 75.

[12] D.A. Tate, J.P. Walton, Phys. Rev. A 59 (1999) 1170. 